\title{
Protective Effects of Remimazolam on Cerebral Ischemia/Reperfusion Injury in Rats by Inhibiting of NLRP3 Inflammasome-Dependent Pyroptosis
}

\author{
Min Shi*, Jing Chen*, Tianxiao Liu (D), Weixin Dai $\mathbb{D}$, Zhan Zhou, Lifei Chen, Yubo Xie (D) \\ Department of Anesthesiology, The First Affiliated Hospital of Guangxi Medical University, Nanning, 53002I, People's Republic of China \\ *These authors contributed equally to this work \\ Correspondence: Yubo Xie, Department of Anesthesiology, The First Affiliated Hospital of Guangxi Medical University, No. 6 Shuang-yong Road, \\ Nanning, 53002I, People's Republic of China, Tel +86 1867795 8208, Email xybdoctor@163.com
}

\begin{abstract}
Introduction: Remimazolam is a novel benzodiazepine $\gamma$-aminobutyric acid A (GABAa) receptor agonist used for sedation and the induction as well as maintenance of general anesthesia. Previous research proved that anesthetic agents acting on GABAa receptor, such as thiopentone, propofol and midazolam, have protective actions for cerebral ischemia/reperfusion (I/R) injury. We here probed into remimazolam for its protective effect and potential mechanism of action against cerebral I/R injury.

Material and Methods: A rat model of middle cerebral artery occlusion (MCAO) with focal transient cerebral $\mathrm{I} / \mathrm{R}$ injury was established and was given tail vein injection of gradient remimazolam $(5,10,20 \mathrm{mg} / \mathrm{kg})$ after $2 \mathrm{~h}$ of ischemia. Following $24 \mathrm{~h}$ of reperfusion, neurological function, brain infarct volume, morphology of cerebral cortical neurons, and expressions of corticocerebral NLRP3, ASC, caspase-1, GSDMD, IL-1 $\beta$ and IL-18 were evaluated.

Results: The results showed that remimazolam could effectively improve the neurological dysfunction, reduce the infarct volume and alleviate the damage of cortical neurons after I/R injury. Notably, the expression of NLRP3 inflammasome pathway was downregulated, suggesting that remimazolam exerted protective actions on $\mathrm{I} / \mathrm{R}$ injury by suppressing pyroptosis with decreased expression and release of inflammatory factors, and the involvement of the NLRP3 inflammasome pathway might be the core during that process. Overall, our results indicate that NLRP3 inflammation is a promising target.

Conclusion: Based on this mechanism, remimazolam may be one of the ideal anesthetic drugs for patients with ischemic stroke. Keywords: remimazolam, NLRP3 inflammasome, ischemia/reperfusion injury, I/R, pyroptosis, inflammation
\end{abstract}

\section{Introduction}

Stroke is one of the primary causes of death in human with an annually increasing mortality. ${ }^{1}$ In most cases, there is a need of surgery, such as stent implantation and cerebrovascular recanalization. ${ }^{2}$ General anesthesia can contribute to lesser stress response and hemodynamics stability during surgery and has become the best choice for critical stroke. However, such management can be potentially risky for perioperative brain injury, ${ }^{3}$ and the use of improper anesthetic agent may cause severe conditions. Given the fact, perioperative brain protection is always been a challenge in anesthesiologists and neurologists, and the selection of optimal anesthetic agent appears to be much more prominent.

Archer et $\mathrm{al}^{4}$ reported that general anesthesia by propofol, isoflurane and sevoflurane could alleviate the ischemic nerve injury in rodents of focal cerebral ischemia, yet the underlying mechanism was not clarified. In studies by Yang et al, ${ }^{5}$ Peng et al, ${ }^{6}$ Shi et al, ${ }^{7}$ Liu et $\mathrm{al}^{8}$ and Jiang et $\mathrm{al}^{9}$ it was also found that the use of propofol, isoflurane, sevoflurane, midazolam and dexmedetomidine contributed to improved outcome of stroke with improved cerebral infarction and $\mathrm{I} / \mathrm{R}$ injury. Inhaled anesthetics are rarely used for stroke due to the possible risk for increased intracranial pressure. Instead, intravenous anesthetics, such as propofol, dexmedetomidine and midazolam, are popular while with certain side effects 
which limit their use in clinic, for example, propofol has significant suppressive effect on circulation, dexmedetomidine is relatively poor in sedation and midazolam leads to a high incidence of delayed awakening from anesthesia.

Remimazolam is a novel benzodiazepine agonist targeting $\gamma$-aminobutyric acid A (GABAa) receptor. It can stabilize hemodynamics while providing rapid anesthesia and awakening, and produce lesser suppressive effect toward breathing. In the meantime, it allows for prolonged infusions without accumulation and the metabolites have no any pharmacological action. Owing to the advantages, remimazolam is promising in clinical applications. ${ }^{10,11}$ GABAergic signaling participates in multiple functions of macrophages, but the underlying mechanism has not been clarified yet. ${ }^{12}$ It was reported that GABA receptor-associated protein, GABARAP, affected nucleotide-binding oligomerization domain-like receptors pyrin domain containing 3 (NLRP3) inflammasome-dependent inflammation via mediating the mitochondrial mass of relevant macrophages. ${ }^{13}$ In addition, higher GABA receptor expression and functions could be protective for the acute injury resulting from transient focal cerebral ischemia. ${ }^{14}$

NLRP3 inflammasome pathway was proven to be vital significant in cerebral $\mathrm{I} / \mathrm{R}$ and following pyroptosis. ${ }^{15,16}$ Specifically, NLRP3 is activated in microglial cells in response to $I / R$ injury, subsequently expresses in neuron and microvascular endothelial cells. ${ }^{17}$ In that way, blocking NLRP3 activation and expression of related proteins in neuron and microglial cells was reported to be an effective approach against focal cerebral $\mathrm{I} / \mathrm{R}$ injury, contributing to improved neurological function score, decreased infarct volume and lighter cerebral edema. ${ }^{18-20}$

We here propose a hypothesis that remimazolam can play protective actions on brain with the involvement of NLRP3 inflammasome pathway, which is important in pyroptosis and further inflammatory cascade to reduce the neuronal damage resulted from I/R. To this end, rat models of MCAO were constructed to study possible effects and corresponding molecular mechanism.

\section{Materials and Methods}

\section{Animals}

Seventy-five male, SPF, Sprague-Dawley rats (7-8 weeks, 250-300 g) were obtained from laboratory animal center of Guangxi Medical University. All rats were allowed to acclimate for 1 week before modeling $\left(22-25^{\circ} \mathrm{C}\right.$, relative humidity $55-$ $60 \%, 12 / 12$ light/dark), with free water and diet. They were fasted and deprived of water, respectively, $12 \mathrm{~h}$ and $4 \mathrm{~h}$ before the experiment. The animal experiment was conducted with the approval of the ethics committee of Guangxi Medical University, strictly following The Guide for Care and Use of Laboratory Animals released by National Institutes of Health.

\section{Materials}

Remimazolam tosilate for injection was purchased from HengRui Medicine Co., Ltd. (Jiangsu, China). Antibodies included anti-NLRP3, anti-ASC, anti-caspase-1, anti-GSDMD, anti- $\beta$-actin, anti-IL-1 $\beta$ and anti-IL-18 antibodies (Abcam and Cell Signaling Technology, USA). Detection kits included SP kit (Zhongshan Golden Bridge Biotechnology, Beijing, China), BCA protein assay kit and hematoxylin-eosin (HE) staining kit (Solaibao Biotechnology Co., Ltd., Beijing, China).

\section{Model Establishment and Grouping}

Rat models of MCAO were constructed following the Longa method. ${ }^{21}$ The right common carotid artery (CCA), external carotid artery (ECA) and internal carotid artery (ICA) were isolated by dissection. A intraluminal filament was placed from ECA to ICA, passing through the bifurcation of CCA. The intraluminal filament reached the intracranial part of ICA to block the right MCA blood flow, with the strand-forward length as (18 \pm 2$) \mathrm{mm}$ from the bifurcation of CCA.

A random method was applied to equally divide the 75 rats into 5 groups: Sham, MCAO, MCAO+ $R_{L}, M C A O+R_{M}$, and $\mathrm{MCAO}+\mathrm{R}_{\mathrm{H}}$. Blood flow blocking was managed $2 \mathrm{~h}$ in all MCAO rats and reperfusion was performed after that by removing the strand. Rats from $M C A O+R_{L}, M C A O+R_{M}$, and $M C A O+R_{H}$ groups were, respectively, assigned to receive additional gradient remimazolam by tail vein injection instantly after reperfusion $(5 \mathrm{mg} / \mathrm{kg}, 10 \mathrm{mg} / \mathrm{kg}, 20 \mathrm{mg} / \mathrm{kg})$. All the rats were sacrificed under anesthesia after $24 \mathrm{~h}$ of reperfusion, and the brain was immediately isolated for further analysis. 


\section{Neurological Function Score}

Five-grade (0-4 points) Longa method was applied to confer a neurological deficit score (NDS) at $2 \mathrm{~h}$ of ischemia and $24 \mathrm{~h}$ of reperfusion, respectively. Grade I (0-point): normal behavior without any neurological deficit; Grade II (1-point): the left front paw fails to fully extend; Grade III (2-point): turn to the left (paralyzed side) while walking; Grade IV (3-point): tip to the left (paralyzed side) while walking; Grade V (4-point): unable spontaneous walking with impairment of consciousness. Models with $2 \mathrm{~h}$ of ischemia scored 1-3 points were included, otherwise excluded from the study.

\section{Triphenyltetrazolium-Chloride (TTC) Staining}

Following model establishment, the rats were decapitated under deep anesthesia and the brain tissue excluding cerebellum, olfactory bulb and lower brain stem was extracted, transferred to PBS solution $\left(0-4^{\circ} \mathrm{C}\right)$ for cleaning and then frozen at $-20^{\circ} \mathrm{C}$ for $20 \mathrm{~min}$. The tissue block was cut to obtain coronal brain slices $(2 \mathrm{~mm})$. Being exposed to $2 \%$ TTC solution, the slices were water-bathed at $37^{\circ} \mathrm{C}$ in the dark for $30 \mathrm{~min}$. Shaking at 5 -min intervals was performed to ensure uniform staining. Subsequently, the stained tissue samples were fixed overnight with $4 \%$ paraformaldehyde, and dried on the following day for photography. Image-ProPlus 6.0 software was run to obtain the infarct area (white) on the tail side, and the infarct volume was calculated according to the slice thickness.

\section{Hematoxylin-Eosin (HE) Staining}

The rats were transcardially perfused with $4 \%$ paraformaldehyde after anesthesia. Intact brain tissue was isolated, fixed, washed, dehydrated, and then processed for transparency and preparation of paraffin-embedded blocks. Coronal sections $(5 \mu \mathrm{m})$ at the posterior optic chiasma were obtained. The sections were then deparaffinized through xylene and ethanol to water, followed by staining with hematoxylin and eosin. Tissue slides were prepared and observed microscopically for morphology of cortical neurons.

\section{Immunohistochemistry (IHC) Staining}

Paraffin tissue sections were prepared and then processed according to the instructions provided by SP kit. In short, the sections were deparaffinized through gradient xylene and ethanol to water, followed by high-temperature antigen repair with sodium citrate. $0.3 \%$ hydrogen peroxide and goat serum were sequentially added for blocking with each 15 min. Afterwards, the tissue samples were consecutively exposed to primary antibodies (NLRP3, GSDMD, ASC, caspase-1, IL-1 $\beta$ and IL-18) for $1 \mathrm{~h}$, biotin-labeled secondary antibody for $15 \mathrm{~min}$, and HRP-conjugated SPTM for 15 min. DAB was applied to develop color and hematoxylin was supplemented for counterstaining. The results were observed microscopically. Negative control was free of primary antibody. Image-Pro Plus 6.0 was operated to perform quantitative analysis. The positive rate was represented by average optical density ( $\mathrm{AOD}=\mathrm{IOD} / \mathrm{Area})$.

\section{Western Blot}

Peripheral cortex $(0.1 \mathrm{~g})$ of infarct area was collected by cryogenic grinding and lysed on ice with $1 \mathrm{~mL}$ lysis (RIPA + PMSF + phosphatase inhibitor) for half an hour. The lysates were cryogenically centrifuged for $15 \mathrm{~min}$. The products were cryogenically centrifuged, and the supernatant was then harvested to determine protein concentration with the BCA method. The protein samples were mixed with $5 \times$ sample buffer at $4: 1$, degenerated by boiling at $100^{\circ} \mathrm{C}$ for 10 min, and then processed by SDS-PAGE. The obtained proteins were loaded to a PVDF membrane by wet-transfer, and then exposed to $5 \%$ skim milk for $1 \mathrm{~h}$ at room temperature to avoid unspecific binding. Primary antibodies, including anti-NLRP3, anti-GSDMD, anti-ASC, anti-caspase-1, anti-IL-1 $\beta$ and anti-IL-18 were added overnight at $4^{\circ} \mathrm{C}$, followed by fluorescent dye-coupled secondary antibody for $1 \mathrm{~h}$ at room temperature. Images were captured by Odyssey system (LI-COR Biosciences), and analyzed on Image $J$ to obtain protein expression. $\beta$-actin acted as the internal control. 


\section{Statistical Analysis}

Data were presented as mean \pm SD and processed on SPSS 25.0 and GraphPad Prism 8.0.2. Data normality was assessed with QQ plots (see the Supplementary Materials). Normally distributed data were analysed with one-way ANOVA followed by SNK-q. Skewed data were analysed with Kruskal-Wallis test followed by Dunn's multiple comparisons. P < 0.05 was considered statistically significant.

\section{Results}

\section{Neurological Function Score}

Sham rats were scored 0 , regarded normal without any neurological deficit. MCAO rats were presented with evident neurological deficits, such as failure to fully extend the left front paw, turning or tipping to the left while walking. In the meantime, they had higher scores after $2 \mathrm{~h}$ of ischemia relative to the Sham group $(p<0.001)$. Moreover, the injection of remimazolam at 5 and $10 \mathrm{mg} / \mathrm{kg}$ after $24 \mathrm{~h}$ of reperfusion contributed to significantly lower scores, demonstrating alleviated neurological disorders ( $p<0.05 ; p<0.01$; Figure 1).

\section{Brain Infarct Volume}

Brain tissue of Sham rats was in uniform red without white infarct area. To the contrary, significant white infarct areas were present in the MCAO group $(p<0.001)$. Following injection of remimazolam, the areas were evidently reduced $(p<0.001)$, which was much more significant at a medium concentration in the MCAO+ $\mathrm{R}_{\mathrm{M}}$ group $(p<0.01$ versus the $\mathrm{MCAO}+\mathrm{R}_{\mathrm{L}}$, and $p<0.001$ versus the $\mathrm{MCAO}+\mathrm{R}_{\mathrm{H}}$; Figure $2 \mathrm{~A}$ and $\mathrm{B}$ ).

\section{Morphology of Ischemic Cortical Neuron}

In the HE staining results, Sham rats showed well-organized neurons, round or cone-shaped cells with intact structure, clear nucleoli and boundaries; in the MCAO group, there was evidence of cell death, including significant neuronal degeneration, loose tissue structure, interstitial edema, swollen neural cells in triangular or irregular shapes, which were

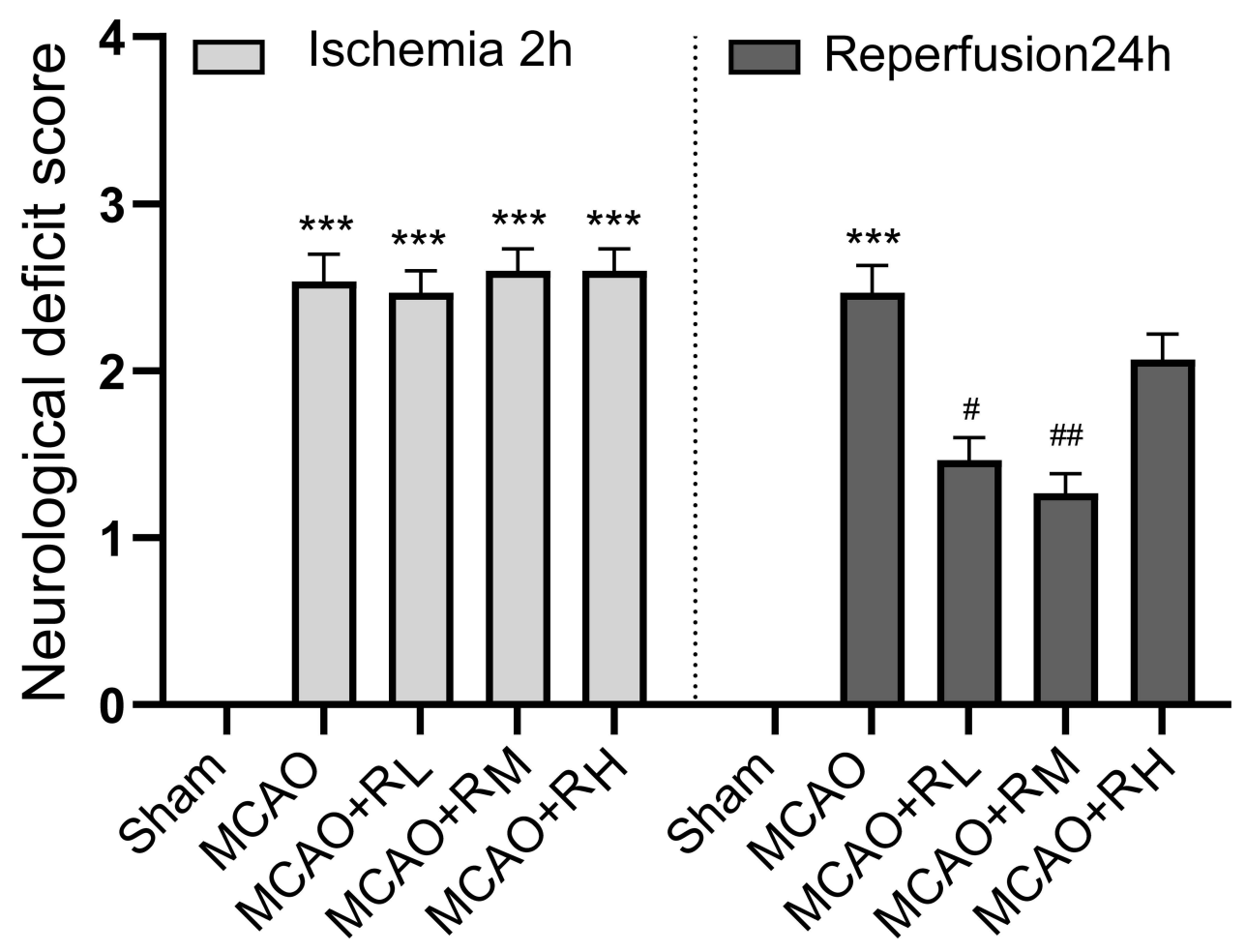

Figure I NDS at $2 \mathrm{~h}$ of ischemia and $24 \mathrm{~h}$ of reperfusion.

Notes: ${ }^{* * *} p<0.001$, compared to Sham group; ${ }^{\#}<<0.05,{ }^{\#} p<0.01$, compared to MCAO group; $n=15$. 

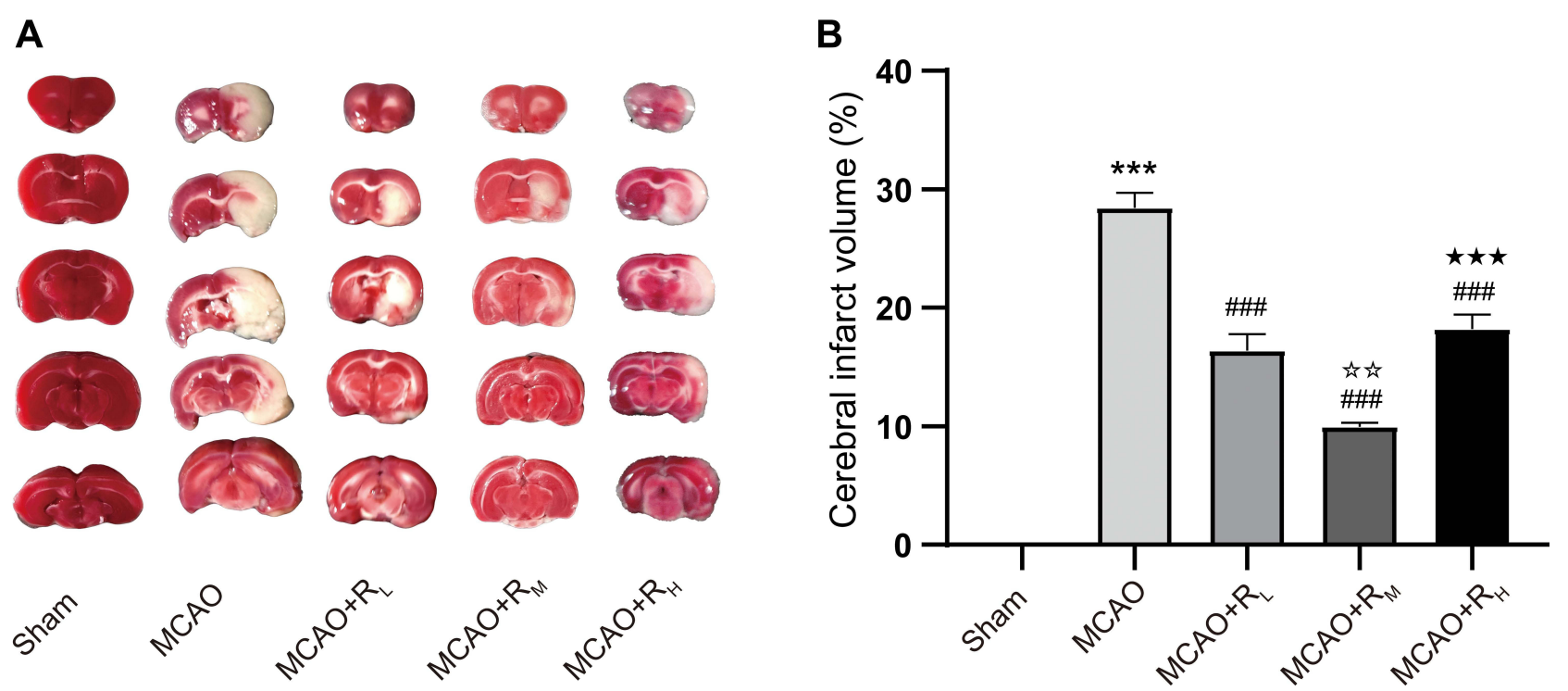

Figure 2 TTC staining for brain infarct volume.

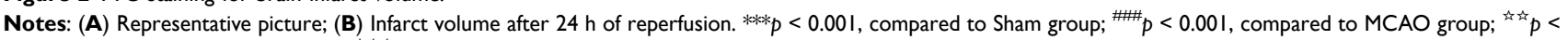
0.01 , compared to $M C A O+R_{L}$ group; ${ }^{\star \star \star} p<0.001$, compared to MCAO+R $R_{M}$ group; $n=5$.

disordered, with nuclear pyknosis and fragmentation. Treatment of remimazolam contributed to significantly alleviated injury (Figure 3).

\section{Brain NLRP3, ASC, Caspase-I, GSDMD, IL-I $\beta$ and IL-I 8 Expression IHC Staining}

Expressions of NLRP3, ASC, caspase-1, GSDMD, IL-1 $\beta$ and IL-18 at the border zone of infarcts were measured by IHC (Figure 4). Relative to the Sham group, higher expressions were present in the MCAO group ( $p<0.001$, Figure 4B-G). Relative to the MCAO group, significant reductions were found in NLRP3, ASC, caspase-1, GSDMD, IL-18 in MCAO $+\mathrm{R}_{\mathrm{L}}\left(p<0.05 ; p<0.01 ; p<0.001\right.$; Figure 4B-E and G), NLRP3, ASC, caspase-1, GSDMD, IL-1 $\beta$ and IL-18 in MCAO+ $\mathrm{R}_{\mathrm{M}}$ $(p<0.001$; Figure $4 \mathrm{~B}-\mathrm{G})$, and NLRP3, ASC, GSDMD, IL-1 $\beta$ and IL-18 in MACO $+\mathrm{R}_{\mathrm{H}}(p<0.05 ; p<0.01 ; p<0.001$; Figure $4 \mathrm{~B}, \mathrm{C}, \mathrm{E}-\mathrm{G})$ groups. Relative to the $\mathrm{MCAO}+\mathrm{R}_{\mathrm{L}}$ group, ASC, GSDMD, IL-1 $\beta$ and IL-18 expressions were significantly decreased in the $\mathrm{MCAO}+\mathrm{R}_{\mathrm{M}}$ group $(p<0.05 ; p<0.01$; Figure $4 \mathrm{C}, \mathrm{E}-\mathrm{G})$. Relative to the $\mathrm{MCAO}+\mathrm{R}_{\mathrm{M}}$ group, ASC, caspase-1, GSDMD and IL-18 were profoundly elevated in the MCAO $+\mathrm{R}_{\mathrm{H}}$ group $(p<0.05, p<0.01$ Figure $4 \mathrm{C}-\mathrm{E}$ and G).

\section{Western Blot}

Figure 5A shows the protein bands of NLRP3, ASC, caspase-1, GSDMD, IL-1 $\beta$ and IL-18 in each group. Higher protein expressions were present in the MCAO group versus the Sham group $(p<0.05 ; p<0.001$; Figure 5B-G). Relative to the MCAO group, significant reductions were found in ASC, caspase-1, IL- $1 \beta$ and IL-18 in MCAO+ $\mathrm{R}_{\mathrm{L}}(p<0.05$; Figure $5 \mathrm{C}, \mathrm{D}$, F and G); NLRP3, ASC, caspase-1, GSDMD, IL-1 $\beta$ and IL-18 in MCAO+ $\mathrm{R}_{\mathrm{M}}(p<0.05 ; p<0.01 ; p<0.001$; Figure 5B-G); and NLRP3, caspase-1 in MACO $+\mathrm{R}_{\mathrm{H}}\left(p<0.05, p<0.01\right.$, Figure 5B and D) groups. Relative to the MCAO+ $\mathrm{R}_{\mathrm{L}}$ group, NLRP3 and caspase- 1 expressions were highly decreased in the MCAO $+\mathrm{R}_{\mathrm{M}}$ group $(p<0.05$; Figure $5 \mathrm{~B}$ and $\mathrm{D})$. Relative to the MCAO $+\mathrm{R}_{\mathrm{M}}$ group, NLRP3 and ASC were profoundly increased in the $\mathrm{MCAO}+\mathrm{R}_{\mathrm{H}}$ group $(p<0.05$; Figure $5 \mathrm{~B}$ and $\mathrm{C})$.

\section{Discussion}

MCAO models are commonly established to mimic the pathological conditions following the onset of middle cerebral artery occlusion. It allows the induction of focal I/R injury and provides a subject view toward relevant functions. ${ }^{22}$ Here, rat models of right MCAO were established to investigate the effect of remimazolam on cerebral $\mathrm{I} / \mathrm{R}$ injury. It is known that focal cerebral I/R injury generally can cause morphological changes and dysfunction of ischemic cortical neurons, 


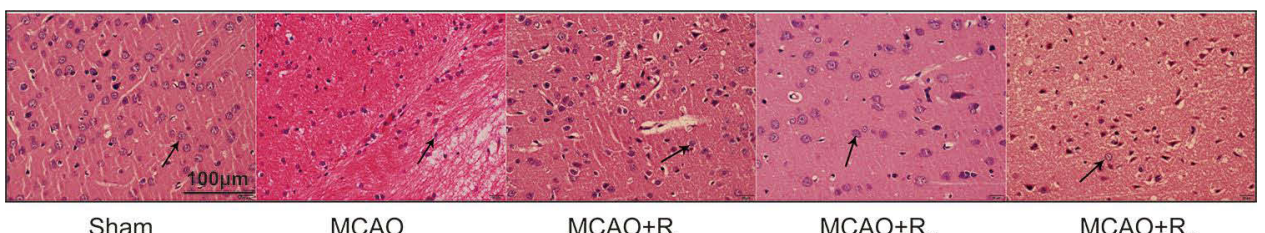

Figure 3 HE staining for morphology of peripheral cortical neuron of cerebral ischemic area (Scale: $100 \mu \mathrm{m}$ ).

Notes: Sham group: well-organized neural cells, with intact structure, large and round nuclei, and clear nucleoli (arrow); MCAO group: irregular, disordered neuralcells, with nuclear pyknosis and fragmentation (arrow); $M C A O+R_{L} / M C A O+R_{M} / M A C O+R_{H}$ : better cell morphology (arrow) than the MCAO group, much more significant in the $M C A O+R_{M}$ group.

and result in cerebral infarction to the most critical degree after $24 \mathrm{~h}^{23}$ The rats here suffered from acute neurological disorders of varying degrees after 24 of reperfusion. TTC and HE staining revealed significant infarct lesions in the MCA blood supply area, in the meantime, pathologically injured or dead neurons were present. Following reperfusion and instant remimazolam injection, great improvements were found in neurological function scores, neuronal injury and brain infarct volume, suggesting the effectiveness of remimazolam in improving the neurological disorders and decreasing the damage to cortical neurons after $\mathrm{I} / \mathrm{R}$ injury.

Cerebral $\mathrm{I} / \mathrm{R}$ injury is a result of a series of complex cascade reactions. Major pathological changes after ischemia include microvascular injury, blood-brain barrier dysfunction, inflammation, oxidative stress/reactive oxygen species increase, and reduced neuronal survival. ${ }^{24}$ During reperfusion process, oxygen radical, cytokine and inflammatory factor will be largely released. In the meantime, there will be an increase in inflammatory cell infiltration because of the increased permeability of blood-brain barrier, thus propagating inflammatory response to cause microvascular blocking and further secondary hypoperfusion. ${ }^{25}$ Inflammation, one of the pathological factors for cerebral I/R injury, has been recognized as a vital part in cerebral ischemic stroke. Both the formation and development of local inflammation are involved in the injury and necrosis of neurons. Consistently, we here found an evident increase in cortical IL-1 $\beta$ and IL18 in the ischemic area after MCAO establishment, which also supported the vital role of inflammation in acute cerebral injury.

Pyroptosis is a type of programmed cell death dependent on cysteinyl aspartate specific proteinase 1 (caspase-1). ${ }^{26,27}$ It is different from apoptosis and shows a close relationship with inflammation. Typical characteristics of pyroptosis are cell swelling, cell membrane blebbing, pore-forming resulting in cell death due to rupture, and outflow of intracellular substances. ${ }^{28}$ Pyroptosis is majorly present in phagocytic cells such as macrophages and neutrophils, and also can be seen in endothelial cells and neurons. ${ }^{29}$

It is established that inflammasome activation is the typical and key part in the regulation of pyroptosis. It can be triggered by varying stimulatory signals involving two patterns: pathogen-associated molecular patterns (PAMP) and danger-associated molecular patterns (DAMP). Research revealed that following the onset of stroke there is typical NLRP3 inflammasome activation triggered by the DAMP pattern. ${ }^{30,31}$ NLRP3 inflammasome is composed of nucleotidebinding oligomerization domain protein-like receptors (NLRs), adaptin including apoptosis-associated speck-like protein containing (ASC) and effector protein such as pro-caspase-1. It participates in regulating inflammatory cascades in multiple diseases, in the meantime, shows a role in pyroptosis by affecting pore-forming protein gasdermin D (GSDMD). ${ }^{32,33}$; The activated NLRP3 will promote pro-caspase-1 to transform into caspase-1 of hydrolysis activity, in turn activating several proinflammatory cytokines including IL-1 $\beta$ and IL-18. ${ }^{34}$ GSDMD is a specific effector molecule involved in pore channel formation and the N-terminal fragment function is the key for the occurrence of pyroptosis. ${ }^{35}$ In the context of caspase-1 activation, GSDMD can be lysed as GSDMD-NT, which affects the permeability of cell membrane, and in turn triggers pyroptosis and release of inflammatory cytokines such as IL- $1 \beta$ and IL$18 .^{36}$

$\mathrm{Fu}$ et $\mathrm{al}^{37}$ reported that neuronal injury was accompanied by activation of NLRP3 inflammasome and strong inflammatory reactions with vast IL-1 $\beta$ and IL-18 released. In this study, IHC and Western blot assays were devised to test cortical expressions of NLRP3, ASC, caspase-1, GSDMD, IL-1 $\beta$ and IL-18. Consistent with the previous studies, 


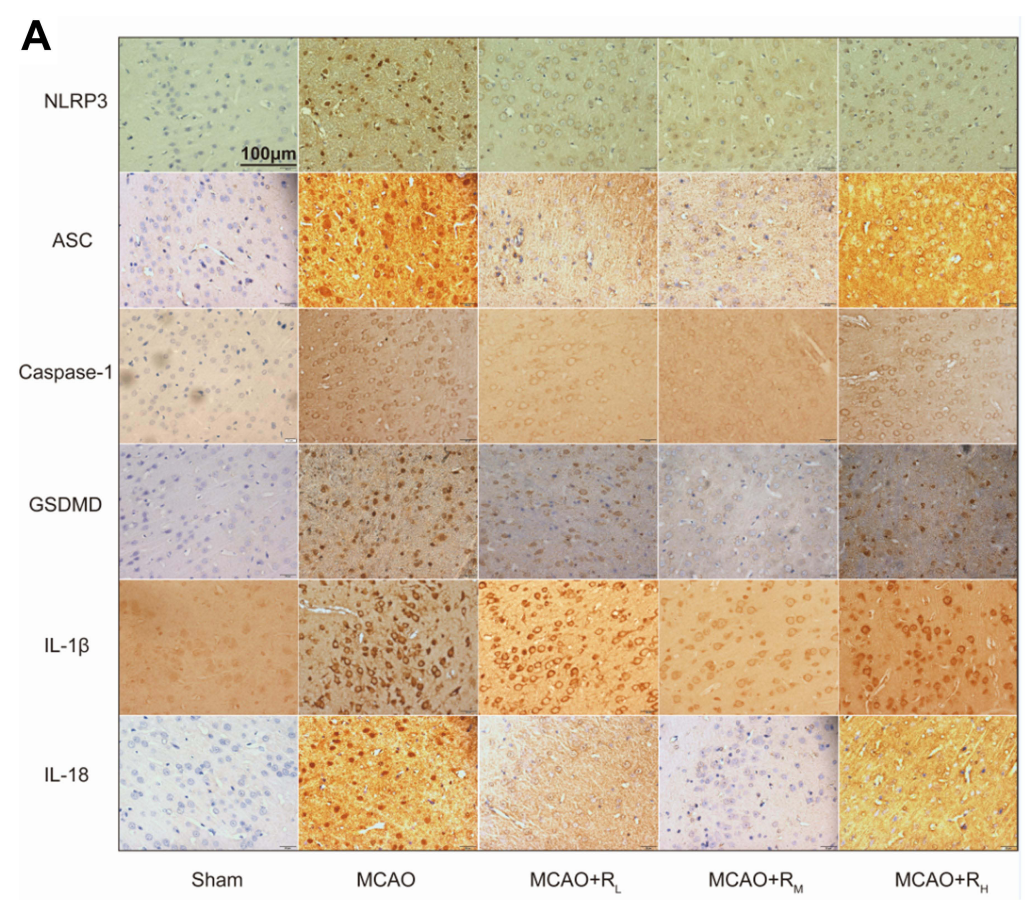

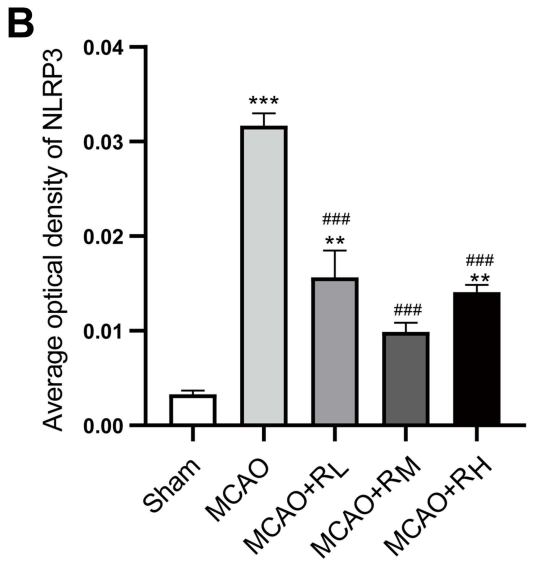

C

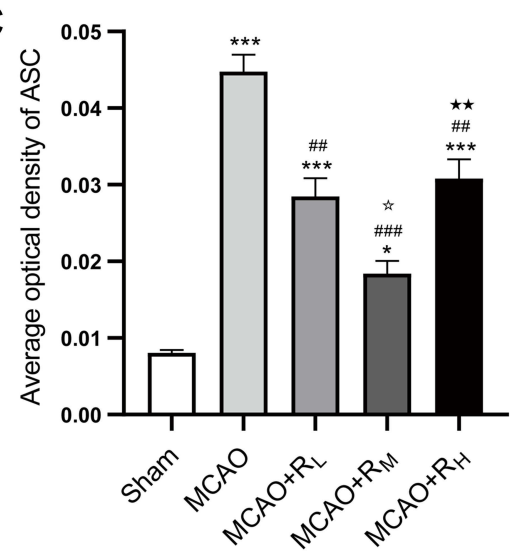

E

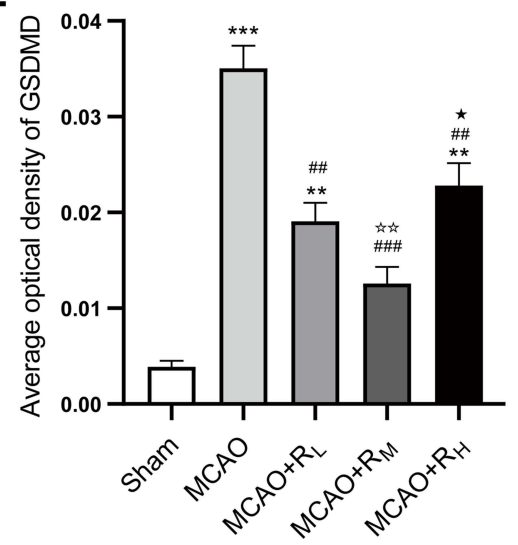

F

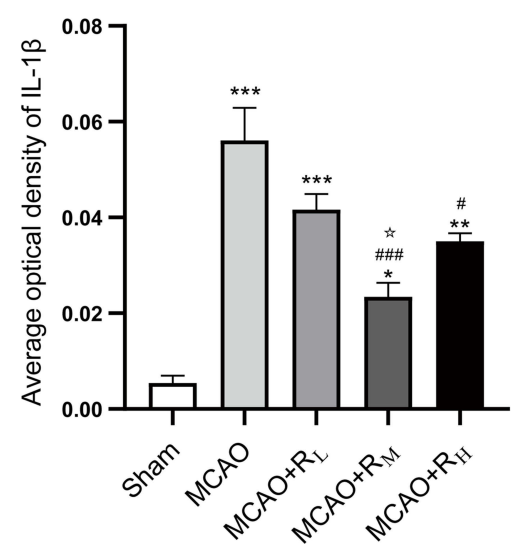

D

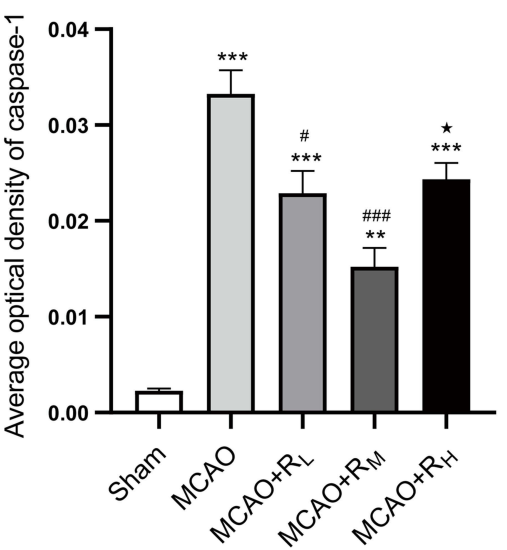

G

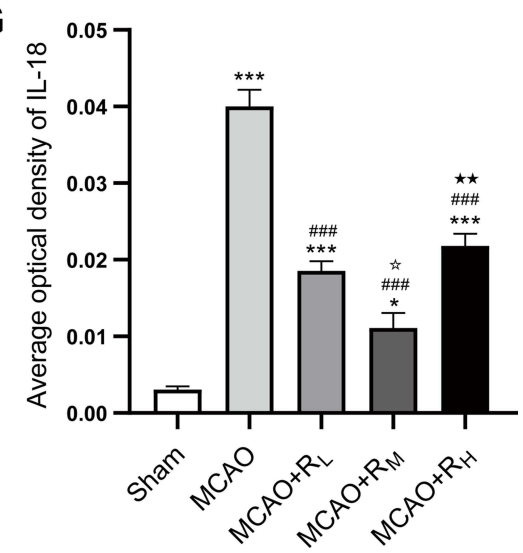

Figure 4 IHC staining for border zone infarcts. (A) Micrographs for NLRP3, ASC, caspase-I, GSDMD, IL-I $\beta$ and IL-I8 at $24 \mathrm{~h}$ of reperfusion (Scale: I00 $\mu \mathrm{m}$ ).

Notes: Positive areas were in dark brown. The Sham group showed low positive expressions, while the MCAO group displayed abundant expressions, which were reduced

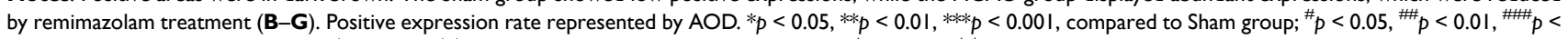
$0.00 \mathrm{I}$, compared to MCAO group; ${ }^{\text {th }} p<0.05$, ${ }^{\star 2} p<0.01$, compared to $M C A O+R_{L}$ group; ${ }^{\star} p<0.05,{ }^{\star} p<0.01$, compared to $M C A O+R_{M}$ group; $n=5$. 
A
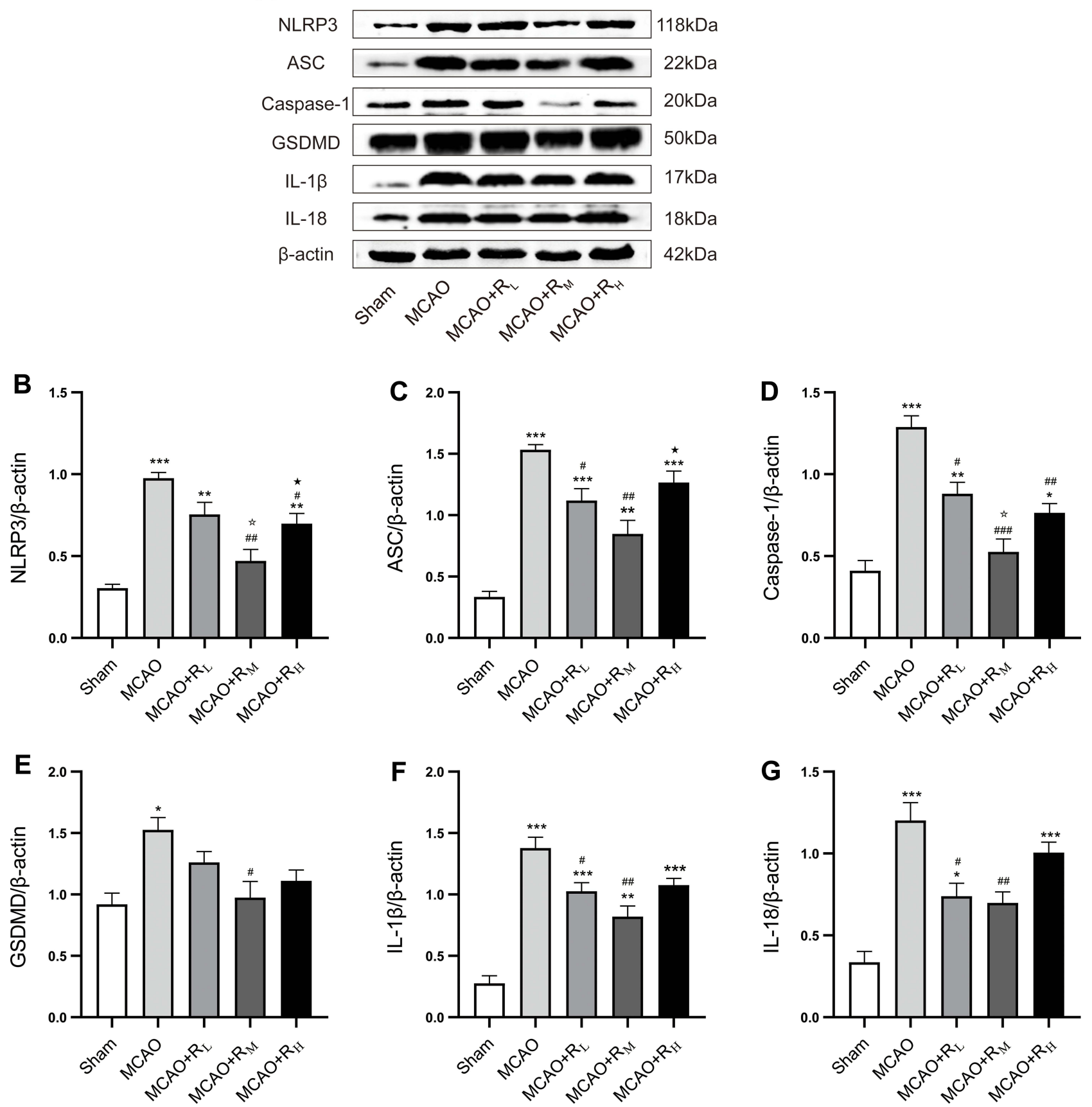

Figure 5 Protein expressions of cortical NLRP3, ASC, caspase-I, GSDMD, IL-I $\beta$ and IL-I8 after remimazolam treatment.

Notes: (A) Representative protein bands; (B-G) Quantitative analysis for protein expression standardized by $\beta$-actin. $* p<0.05$, $* * p<0.01$, $* * * p<0.00$ I, compared to Sham group; ${ }_{p} p<0.05,{ }^{\#} p<0.01,{ }^{\# \#} p<0.001$, compared to MCAO group; ${ }^{\star} p<0.05$, compared to $M C A O+R_{L}$ group; ${ }^{\star} p<0.05$, compared to $M C A O+R_{M}$ group; $n=5$.

NLRP3 and ASC, vital components of NLRP3 inflammasome, were highly up-regulated following the onset of transient I/R injury, suggestive of NLRP3 inflammasome activation. The expressions of caspase-1 and GSDMD were also evidently increased resulting in the incidence of pyroptosis, and strong inflammatory reactions concurrently occurred with more activated inflammatory factors IL-1 $\beta$, IL-18. Studies have revealed that inhibition of NLRP3-mediated pyroptosis might have neuroprotective effect. ${ }^{38-40}$ Besides, neuronal injury could be alleviated after application of NLRP3 inhibitor MCC95 and caspase-1 inhibitor Ac-YUAD-CMK. ${ }^{41}$ NLRP3-mediated pyroptosis and inflammatory cascade may be crucially potential targets for intervention in ischemic stroke. Notably, expressions of the mentioned 
factors such as NLRP3, caspase-1, GSDMD IL-1 $\beta$ and IL-18 displayed a significant decreasing trend after remimazolam application. It demonstrated that remimazolam, to some extent, could suppress NLRP3-mediated pyroptosis and further inflammatory cascade. The above results were consistent with the morphological and functional changes of neurons in rats, altogether implied the possible mechanism of action of remimazolam, by which remimazolam suppresses NLRP3 inflammasome-induced pyroptosis to reduce activation and release of inflammatory factors and subsequent inflammatory cascade, finally alleviating cerebral I/R injury and improving neuronal morphology and function.

Perioperative organ protection is an important topic in Anesthesiology. Remimazolam has a broader application prospect because of its special advantages compared with existing GABA receptor agonists. The study of the effect of remimazolam on cerebral I/R injury is in line with the theme of organ protection, and has certain practical significance for guiding clinical medication in patients with ischemic stroke. Although we found and preliminarily verified the important role of NLRP3 inflammasome pathway in the protection of remimazolam on cerebral $\mathrm{I} / \mathrm{R}$ injury in rats, considering the differences between animal models and clinical practice, as well as the ethical limitations of clinical studies, it is necessary to evaluate the safety and effectiveness of remimazolam in patients with ischemic stroke through high-quality, large-sample and multicenter prospective studies. In addition, the concentration gradients of $5 \mathrm{mg} / \mathrm{kg}$, $10 \mathrm{mg} / \mathrm{kg}$ and $20 \mathrm{mg} / \mathrm{kg}$ were selected according to the $\mathrm{ED}_{50}$ of remimazolam in rats, ${ }^{42}$ but the protective effect in this experiment was not dose-dependent. Moreover, this study did not use more methods to activate or block related signal pathways for further verification. Therefore, the appropriate dose and exact neuroprotective mechanism of remimazolam still need to be carried out in more in-depth researches in the future.

\section{Conclusion}

To sum up, this research identified the role of NLRP3 inflammasome-dependent pyroptosis in occurrence of cerebral I/R injury, which cooperates with secondary inflammatory cascade to cause morphological changes and dysfunction of cerebral cortical neurons. Remimazolam was proven to have protective actions for cerebral I/R injury in rats, since it could reduce neuronal injury and cerebral infarct as well as improve neurological function. The NLRP3 inflammasomedependent pyrolysis pathway may play a vital significance regulatory role in the mechanism about neuroprotective of remimazolam.

\section{Abbreviations}

$\mathrm{I} / \mathrm{R}$, ischemia/reperfusion; MCAO, middle cerebral artery occlusion; GABAa, $\gamma$-aminobutyric acid A; NLRP3, nucleotide-binding oligomerization domain-like receptors pyrin domain containing 3; CCA, common carotid artery; ECA, external carotid artery; ICA, internal carotid artery; NDS, neurological deficit score; TTC, triphenyltetrazolium-chloride; HE, hematoxylin-eosin; IHC, immunohistochemistry; AOD, average optical density.

\section{Acknowledgments}

This work was supported by the Key Research and Development program of Guangxi (No. AB18221031), Natural Science Foundation of Guangxi (No. 2020GXNSFDA238025), and National Natural Science Foundation of China (No. 81373498). All authors made substantial contributions to this study. We are grateful to the staff involved in this research. Min Shi, Jing Chen and Tianxiao Liu are co-First authors for this study.

\section{Disclosure}

The authors report no conflicts of interest in this work.

\section{References}

1. Moraga P; GBD 2016 Causes of Death Collaborators. Global, regional, and national age-sex specific mortality for 264 causes of death, 1980 2016: a systematic analysis for the Global Burden of Disease Study 2016. Lancet. 2017;390(10100):1151-1210. doi:10.1016/s0140-6736(17) 32152-9

2. Wu S, Wu B, Liu M, et al. Stroke in China: advances and challenges in epidemiology, prevention, and management. Lancet Neurol. 2019;18 (4):394-405. doi:10.1016/s1474-4422(18)30500-3 
3. Bilotta F, Gelb AW, Stazi E, Titi L, Paoloni FP, Rosa G. Pharmacological perioperative brain neuroprotection: a qualitative review of randomized clinical trials. Br J Anaesth. 2013;110(Suppl 1):i113-20. doi:10.1093/bja/aet059

4. Archer DP, Walker AM, McCann SK, Moser JJ, Appireddy RM. Anesthetic neuroprotection in experimental stroke in rodents: a systematic review and meta-analysis. Anesthesiology. 2017;126(4):653-665. doi:10.1097/aln.0000000000001534

5. Yang CY, Liu SY, Wang HY, et al. Neuroprotection by propofol post-conditioning: focus on PKM $/$ KCC2 pathway activity. Cell Mol Neurobiol. 2018;38(3):691-701. doi:10.1007/s10571-017-0530-0

6. Peng L, Yin J, Wang S, et al. TGF- $32 /$ Smad3 signaling pathway activation through enhancing VEGF and CD34 ameliorates cerebral ischemia/ reperfusion injury after isoflurane post-conditioning in rats. Neurochem Res. 2019;44(11):2606-2618. doi:10.1007/s11064-019-02880-8

7. Shi CX, Jin J, Wang XQ, et al. Sevoflurane attenuates brain damage through inhibiting autophagy and apoptosis in cerebral ischemia-reperfusion rats. Mol Med Rep. 2020;21(1):123-130. doi:10.3892/mmr.2019.10832

8. Liu JY, Guo F, Wu HL, Wang Y, Liu JS. Midazolam anesthesia protects neuronal cells from oxidative stress-induced death via activation of the JNK-ERK pathway. Mol Med Rep. 2017;15(1):169-179. doi:10.3892/mmr.2016.6031

9. Jiang L, Hu M, Lu Y, Cao Y, Chang Y, Dai Z. The protective effects of dexmedetomidine on ischemic brain injury: a meta-analysis. $J$ Clin Anesth. 2017;40:25-32. doi:10.1016/j.jclinane.2017.04.003

10. Keam SJ. Remimazolam: first Approval. Drugs. 2020;80(6):625-633. doi:10.1007/s40265-020-01299-8

11. Schüttler J, Eisenried A, Lerch M, Fechner J, Jeleazcov C, Ihmsen H. Pharmacokinetics and pharmacodynamics of remimazolam (CNS 7056) after continuous infusion in healthy male volunteers: part I. pharmacokinetics and clinical pharmacodynamics. Anesthesiology. 2020;132(4):636-651. doi:10.1097/aln.0000000000003103

12. Xia Y, He F, Wu X, et al. GABA transporter sustains IL-1ß production in macrophages. Science Advances. 2021;7(15). doi:10.1126/sciadv.abe9274

13. Zhang Z, Xu X, Ma J, et al. Gene deletion of gabarap enhances Nlrp3 inflammasome-dependent inflammatory responses. J Immunol. 2013;190 (7):3517-3524. doi:10.4049/jimmunol.1202628

14. Huang L, Li Q, Wen R, et al. Rho-kinase inhibitor prevents acute injury against transient focal cerebral ischemia by enhancing the expression and function of GABA receptors in rats. Eur J Pharmacol. 2017;797:134-142. doi:10.1016/j.ejphar.2017.01.021

15. Bergsbaken T, Fink SL, Cookson BT. Pyroptosis: host cell death and inflammation. Nat Rev Microbiol. 2009;7(2):99-109. doi:10.1038/ nrmicro2070

16. Li F, Xu D, Hou K, et al. Pretreatment of Indobufen and aspirin and their combinations with clopidogrel or ticagrelor alleviates inflammasome mediated pyroptosis via inhibiting NF-kB/NLRP3 pathway in ischemic stroke. J Neuroimmune Pharmacol. 2021;16:835-853. doi:10.1007/s11481020-09978-9

17. Gong Z, Pan J, Shen Q, Li M, Peng Y. Mitochondrial dysfunction induces NLRP3 inflammasome activation during cerebral ischemia/reperfusion injury. J Neuroinflammation. 2018;15(1):242. doi:10.1186/s12974-018-1282-6

18. Ye Y, Jin T, Zhang X, et al. Meisoindigo protects against focal cerebral ischemia-reperfusion injury by inhibiting NLRP3 inflammasome activation and regulating microglia/macrophage polarization via TLR4/NF-kB signaling pathway. Front Cell Neurosci. 2019;13:553. doi:10.3389/ fncel.2019.00553

19. Liu H, Wu X, Luo J, et al. Adiponectin peptide alleviates oxidative stress and NLRP3 inflammasome activation after cerebral ischemia-reperfusion injury by regulating AMPK/GSK-3ß. Exp Neurol. 2020;329:113302. doi:10.1016/j.expneurol.2020.113302

20. Franke M, Bieber M, Kraft P, Weber ANR, Stoll G, Schuhmann MK. The NLRP3 inflammasome drives inflammation in ischemia/reperfusion injury after transient middle cerebral artery occlusion in mice. Brain Behav Immun. 2021;92:223-233. doi:10.1016/j.bbi.2020.12.009

21. Longa EZ, Weinstein PR, Carlson S, Cummins R. Reversible middle cerebral artery occlusion without craniectomy in rats. Stroke. 1989;20 (1):84-91. doi:10.1161/01.str.20.1.84

22. Aspey BS, Taylor FL, Terruli M, Harrison MJ. Temporary middle cerebral artery occlusion in the rat: consistent protocol for a model of stroke and reperfusion. Neuropathol Appl Neurobiol. 2000;26(3):232-242. doi:10.1046/j.1365-2990.2000.00221.x

23. Derugin N, Wendland M, Muramatsu K, et al. Evolution of brain injury after transient middle cerebral artery occlusion in neonatal rats. Stroke. 2000;31(7):1752-1761. doi:10.1161/01.str.31.7.1752

24. Thiankhaw K, Chattipakorn N, Chattipakorn SC. The effects of hyperbaric oxygen therapy on the brain with middle cerebral artery occlusion. J Cell Physiol. 2021;236(3):1677-1694. doi:10.1002/jcp.29955

25. Fernández-López D, Faustino J, Daneman R, et al. Blood-brain barrier permeability is increased after acute adult stroke but not neonatal stroke in the rat. J Neurosci. 2012;32(28):9588-9600. doi:10.1523/jneurosci.5977-11.2012

26. Frank D, Vince JE. Pyroptosis versus necroptosis: similarities, differences, and crosstalk. Cell Death Differ. 2019;26(1):99-114. doi:10.1038/ s41418-018-0212-6

27. Cookson BT, Brennan MA. Pro-inflammatory programmed cell death. Trends Microbiol. 2001;9(3):113-114. doi:10.1016/s0966-842x(00)01936-3

28. Vande Walle L, Lamkanfi M. Pyroptosis. Curr Biol. 2016;26(13):R568-R572. doi:10.1016/j.cub.2016.02.019

29. Lossi L, Castagna C, Merighi A. Neuronal cell death: an overview of its different forms in central and peripheral neurons. Methods Mol Biol. 2015;1254:1-18. doi:10.1007/978-1-4939-2152-2_1

30. Guo Z, Yu S, Chen X, Ye R, Zhu W, Liu X. NLRP3 is involved in ischemia/reperfusion injury. CNS Neurol Disord Drug Targets. 2016;15 (6):699-712. doi:10.2174/1871527315666160321111829

31. Xue Y, EnosiTuipulotu D, Tan WH, Kay C, Man SM. Emerging activators and regulators of inflammasomes and pyroptosis. Trends Immunol. 2019;40(11):1035-1052. doi:10.1016/j.it.2019.09.005

32. Sun R, Peng M, Xu P, et al. Low-density lipoprotein receptor (LDLR) regulates NLRP3-mediated neuronal pyroptosis following cerebral ischemia/ reperfusion injury. $J$ Neuroinflammation. 2020;17(1):330. doi:10.1186/s12974-020-01988-x

33. Zhao S, Li X, Wang J, Wang H. The role of the effects of autophagy on NLRP3 inflammasome in inflammatory nervous system diseases. Front Cell Dev Biol. 2021;9:657478. doi:10.3389/fcell.2021.657478

34. Duez H, Pourcet B. Nuclear receptors in the control of the NLRP3 inflammasome pathway. Front Endocrinol. 2021;12:630536. doi:10.3389/ fendo.2021.630536

35. Hu JJ, Liu X, Xia S, et al. FDA-approved disulfiram inhibits pyroptosis by blocking gasdermin D pore formation. Nat Immunol. 2020;21 (7):736-745. doi:10.1038/s41590-020-0669-6 
36. Liu X, Zhang Z, Ruan J, et al. Inflammasome-activated gasdermin D causes pyroptosis by forming membrane pores. Nature. 2016;535 (7610):153-158. doi:10.1038/nature18629

37. Fu Q, Wu J, Zhou XY, et al. NLRP3/Caspase-1 pathway-induced pyroptosis mediated cognitive deficits in a mouse model of sepsis-associated encephalopathy. Inflammation. 2019;42(1):306-318. doi:10.1007/s10753-018-0894-4

38. An P, Xie J, Qiu S, et al. Hispidulin exhibits neuroprotective activities against cerebral ischemia reperfusion injury through suppressing NLRP3-mediated pyroptosis. Life Sci. 2019;232:116599. doi:10.1016/j.1fs.2019.116599

39. Lu LQ, Tian J, Luo XJ, Peng J. Targeting the pathways of regulated necrosis: a potential strategy for alleviation of cardio-cerebrovascular injury. Cell Mol Life Sci. 2021;78(1):63-78. doi:10.1007/s00018-020-03587-8

40. Cao X, Wang Y, Gao L. CHRFAM7A overexpression attenuates cerebral ischemia-reperfusion Injury via inhibiting microglia pyroptosis mediated by the NLRP3/Caspase-1 pathway. Inflammation. 2021;44(3):1023-1034. doi:10.1007/s10753-020-01398-4

41. Swanson KV, Deng M, Ting JP. The NLRP3 inflammasome: molecular activation and regulation to therapeutics. Nat Rev Immunol. 2019;19 (8):477-489. doi:10.1038/s41577-019-0165-0

42. Liu Y, Xu X, Xie J, et al. Design, synthesis, and biological evaluation of novel CNS 7056 derivatives as sedatives in rats and rabbits. Chem Biol Drug Des. 2016;88(1):38-42. doi:10.1111/cbdd.12731

\section{Publish your work in this journal}

Drug Design, Development and Therapy is an international, peer-reviewed open-access journal that spans the spectrum of drug design and development through to clinical applications. Clinical outcomes, patient safety, and programs for the development and effective, safe, and sustained use of medicines are a feature of the journal, which has also been accepted for indexing on PubMed Central. The manuscript management system is completely online and includes a very quick and fair peer-review system, which is all easy to use. Visit http://www.dovepress.com/testimonials.php to read real quotes from published authors.

Submit your manuscript here: https://www.dovepress.com/drug-design-development-and-therapy-journal 